\title{
Sarcoïdose épididymaire
}

\author{
Ba M.*, Syllac.*, Gueyes M.*, Ndoye A.*, Wotto-Gaye G.**, Fall A.*, Terolbe I.*, \\ NDIAYE P.D.**, MENSAH A.* \\ * Clinique Urologique ; ** Laboratoire d'Anatomie Pathologique - \\ C.H.U Aristide Le Dantec, Avenue Pasteur, Dakar, Sénégal
}

\section{RESUME}

Les auteurs soulignent le polymorphisme clinique de la sarcoïdose et rapportent un cas de localisation épididymaire. Du point de vue étiopathogénique plusieurs hypothèses sont avancées (mycobactéries, facteurs immunologiques, facteurs génétiques, virus). La race noire apparaît plus touchée que la race blanche.

Le traitement chirurgical repose sur la chirurgie d'exérèse que nous avons réalisée sur le nodule épididymaire. Pour le traitement médical nous avons opté pour la chloroquine à la dose de $300 \mathrm{mg} / \mathrm{j}$. Après 4 ans d'évolution l'état du patient est stabilisé.

Mots-clés : épididyme, sarcoïdose.

\section{INTRODUCTION}

La sarcoïdose ou maladie de Besnier Boeck Schaumann (BBS) est une maladie de système atteignant surtout les poumons et la peau, la localisation urogénitale étant rare. Plusieurs hypothèses étiopathogéniques sont avancées, les manifestations cliniques sont polymorphes d'où l'intérêt de préciser les critères diagnostiques. Nous rapportons un cas de sarcoïdose épididymaire.

\section{OBSERVATION}

Mr A.G., 41 ans, de race noire, se plaignait d'algies épididymo-testiculaires évoluant par poussées depuis 10 ans. Les antécédents notaient une rhinorrhée provoquant parfois une obstruction nasale ainsi qu'une injection intra-épididymaire de corticoïdes n'ayant pas entraîné d'amélioration. Il n'existait pas de troubles urinaires.

L'examen physique objectivait :

- une rhinorrhée séro-muqueuse,

- un nodule épididymaire gauche de $1 \mathrm{~cm}$ de diamètre, céphalique, dur, sensible,

- une vingtaine de nodules sous cutanés, fermes, indolores, non prurigineux, mobiles par rapport au plan superficiel, de 0,5 à $2 \mathrm{~cm}$ de diamètre disséminés au niveau du thorax et des membres.

Les examens complémentaires révèlaient :

- des opacités pulmonaires diffuses périhilaires à prédominance gauche,

- une anémie normochrome normocytaire,

- une hyperuricémie à $71 \mathrm{mg} / 1$,

- une recherche de BK dans les urines négative,

Le reste du bilan biologique était normal.

L'examen anatomo-pathologique réalisé après exérése $d u$ nodule épididymaire et d'un nodule sous cutané était en faveur d'une tuberculose. 
Sur la base de ce diagnostic un traitement antituberculeux de 6 mois était institué. L'absence d'amélioration,l'apparition de nouveaux nodules sous cutanés, la persistance des manifestations ORL et des images radiologiques ont amené à de nouvelles biopsies de l'épididyme, de la muqueuse nasale et des nodules sous cutanés. L'examen anatomo-pathologique révèlait un envahissement de l'épididyme et des régions sous cutanées par une prolifération folliculaire granulomateuse faite de cellules géantes multinuclées type Langhans ou à corps étrangers au sein de ces amas. A la périphérie, il existait une discrète couronne lymphocytaire. Les follicules présentaient un remaniement fibreux. Il n'a pas été objectivé de nécrose caséeuse sur tous les prélèvements examinés. L'anatomo-pathologiste concluait à une sarcoïdose cutanée et surtout épididymaire en lieu et place de la tuberculose.

Le traitement antituberculeux était alors arrêté au profit d'un traitement à la chloroquine à la dose de $300 \mathrm{mg} /$ jour.Ce traitement a été poursuivi 3 ans avec surveillance ophtalmologique. L'évolution a été marquée par la régression nette des poussées nodulaires, la disparition de la rhinorrhée, de l'obstruction nasale, des algies épididymo-testiculaires et des opacités radiologiques pulmonaires. Après 4 ans d'évolution, son état est stabilisé.

\section{DISCUSSION}

Lors de la 7ème conférence internationale sur la sarcoïdose en 1976 [8] les éléments suivants sont retenus : "La sarcoïdose est une granulomatose multisystémique touchant le plus souvent l'adulte jeune, et se manifeste habituellement par des adénopathies hilaires bilatérales, un infiltrat pulmonaire et des lésions cutanées ou oculaires.Le diagnostic est affirmé avec d'autant plus de sécurité que les éléments cliniques et radiologiques sont confirrnés par la mise en évi- dence d'un granulome épithélioïde sans nécrose caséeuse dans plus d'un organe ou un test de KVEIM positif (test de diagnostic). Les anomalies immunologiques consistent en une altération de l'immunité à médiation cellulaire et une augmentation (ou des anomalies) des immunoglobulines. Il peut exister une hypercalciurie avec ou sans hypercalcémie. L'évolution et le pronostic sont parfois en rapport avec le mode de début : un début aigu associé à un érythème noueux fait prévoir une évolution courte et une guérison spontanée alors qu'un début progressif peut se compliquer d'une fibrose irréversible. Les glucocorticoïdes atténuent les symptômes et suppriment l'inflammation et la formation du granulome".

La rareté de la localisation épididymaire de la sarcoïdose est rapportée par la plupart des auteurs [11, 13, 14] (Tableau 1). L'incidence de la sarcoïdose urogénitale est faible et estimée à moins de $5 \%$ sur des études anatomopathologiques nécropsiques [11, 15]. Cette incidence est sûrement sous évaluée dans nos régions du fait du polymorphisme clinique et du sous équipement de certains centres. Au Sénégal, les localisations cutanées et pulmonaires ont été rapportées chez six patients par Ayad [1] et Marchand [10].

La race noire de notre patient rejoint l'incidence 78 à $90 \%$ constatée dans cette population $[15,13]$. La maladie de BBS a longtemps été considérée comme une affection de l'adulte [8] en réalité elle survient à tout âge même pour l'atteinte épididymaire où il a été rapporté le cas d'un enfant de 2 ans [11].

Sur le plan étiopathogénique, des hypothèses ont été avancées incrimant le rôle éventuel des mycobactéries [8], des facteurs immunologiques [7], des facteurs génétiques $[4,12]$ ou encore les virus et d'autres micro-organismes [8].

Granulomatose multisystémique, tous les organes peuvent être atteints avec une prédilection pour les localisations endothora- 
ciques [6]. Les lésions cutanées s'observent dans 10 à $35 \%$ des cas tandis que les manifestations oto-rhinolaryngologiques s'observent dans 5 à $7 \%$ des cas [17].

La symptomatologie clinique dans la sarcoidose épididymaire ne présente aucune spécificité. L'atteinte bilatérale est rare et rapportée chez moins de $1 \%$ des patients présentant une localisation épididymaire [13, 14] avec un retentisement variable sur la fertilité. Une localisation testiculaire pose le problème de diagnostic différentiel avec un cancer du testicule [9]. Mc Williams [11] rapporte 1 cas diagnostiqué après orchidectomie pour suspicion de cancer.

Certains tests et examens à visée diagnostique peuvent être pratiqués :

- le test de KVEIM SILTZBACH qui consiste en l'injection intradermique d'extraits cellulaires sarcoïdiens.Ce test est positif dans près de $80 \%$ des cas quand il s'agit d'une sarcoïdose récente [17],

- l'IDR à la tuberculine qui montre une anergie à la tuberculine dans 70 à $90 \%$ des cas [16],

- le taux sérique de l'enzyme de conversion de l'angiotensine (ECA) dont l'élévation non spécifique dans la sarcoïdose a une valeur dans la surveillance évolutive de la maladie $[3,6]$.

Le diagnostic de sarcoïdose a été posé sur la conjonction d'éléments cliniques et paracliniques dont aucun n'est spécifique. Il s'agit bien d'une maladie à score pour laquelle plusieurs items sont définis $[5,7]$ :

- lésion histologique faite d'un granulome épithélioïde sans lésion caséeuse étendue,

- atteinte de plusieurs organes ou systèmes excluant ainsi les granulomatoses de même type à topographie uniquement régionale,

- particularités immunologiques caractérisées par une mobilisation et une activation lymphocytaire au niveau des différents sites de la maladie,
- absence de cause démontrée excluant ainsi les granulomatoses secondaires à des facteurs microbiologiques.

Le diagnostic repose donc sur la conjonction de ces items, le premier étant formellement exigible et associé de façon variable à deux ou trois autres $[3,5,6]$.

Le but du traitement est de promouvoir les regressions des symptômes en créant le minimum d'effets secondaires. Nous avons opté pour la chloroquine qui agit en stabilisant les membranes lysosomiales des macrophages et prévient la transcription de l'ADN des lymphocytes par interposition [17]. La posologie est de 200 à $300 \mathrm{mg} / \mathrm{j}$ avec nécessité d'une surveillance ophtalmologique à la recherche d'une rétinite. Les rechutes sont possibles à l'arrêt du traitement [7] et nous avons été amené à poursuivre ce traitement pendant 3 ans.

Les moyens thérapeutiques proposés dans la sarcoïdose sont en fait variés, l'étiologie n'étant pas connue. Il est possible d'utiliser des corticoïdes par voie générale, des anti inflammatoires non stéroïdiens, des antimitotiques, ou d'autres médicaments dont l'efficacité en clinique humaine peut être discutée [17]. La complexité de l'affection fait qu'il existe plusieurs schémas thérapeutiques, la chirurgie étant essentiellement une chirurgie d'exérèse. L'abstention thérapeutique peut être de mise avec nécessité d'une surveillance clinique régulière, du fait d'une éventuelle régression spontanée de la maladie [14].

\section{REFERENCES}

1. AYAD M., STROBEL M., HANE A., NDIAYE B., MARCHAND J.P., KANE P.A. : Sarcoïdose à double localisation pulmonaire et cutanée. Dakar Médical, 1980, 25, 1, 46-50.

2. BARBOTIN M., DERRIEN J.P., RABIER R., BOBIN P.,COUTURIER Y. : Sarcoïdose pulmonaire : cinq cas sénégalais. Bull. Soc. Méd. Afr. Nre. Lgue. Frse. 1972, 22, 17103-107.

3. BATTESTI J.P. : Critéres de diagnostic de la sarcoìdose. Presse Med., 1981, 10, 673-674. 
4. BRENITZ E.A., STROM B.L. : Epidemiology of sarcoidosis. Epidemiologic Reviews 1983, 5, 124156.

5. CHRETIEN J. : Sarcoïdose : Quelques rappels nosologiques et sémantiques. Presse Med. 1987, 16, 357-358.

6. CHRETIEN J., BAGLIN J.Y. : Sarcoïdose : bases du diagnostic et rappels des classifications Rev. Prat. 1983, 33, 39, 2027-2035.

7. CHRYSTAL R.G. \& Coll. : Pulmonary sarcoidosis, a disease characterized and perpetuated by actived lung T Iymphocytes Ann. Intern. Med. 1981, 94, 73-94.

8. GODARD P.H., MICHELL F.B. : Sarcoïdose. Encycl. Med. Chir, Paris, Poumon, 6039 C10, 4-1984.

9. GROSS A.J., HEINZER H. : Unsual differential diagnosis of testis tumor: intrascrotal sarcoïdosis. J. Urol. 1992, 147, 1112-1114.

10. MARCHAND J.P., NDIAYE B., ARNOLD J. : Sarcoïdose cutanée chez une sénégalaise. Bull. Soc. Med. Afr. Nre. Lgue. Frse 1976, 21, 140.

11. Me WILLIAMS W.A, ABRAMOWITZ L., TIAMSON E. : Epididymal sarcoïdosis, case report and review. J. Urol. 1983, 130, 1201-1203.

12. NAOKI Y., YOSHIKAZU H. : Bilateral epididymal sarcoïdosis. Scand. J. Uro. Nephrol. 1992, 26, 301303.

13. RITCHIE A.W.S., HINDMARCH J.R. : Bilateral epididymal sarcoidosis. Brit. J. Urol 1983, 55, 240.

14. RYAN D.M \& al : Epididymal sarcoidosis. J. Urol. 1993, 149, 134-136.

15. SARTWELL P.E. : Racial difference in sarcoidosis. Ann. N.Y. Acad. Sci. 1976, 278, 368-370.

16. TURIAF J., MENAULT M., BATTESTI J.P. : Recherche sur les manifestations immunologiques de la sarcoïdose Ann. Med. Int. 1987, 121, 117134.

17. VERNAY VAISSE CH., BERBIS PH., PRIVAT Y. : Sarsoidose. Encycl. Med. Chir (Paris-France), dermatologie, 12515, A10, 41988, 12P.

\section{ABSTRACT}

\section{Epididymal sarcoidosis}

M. BA, C. Sylla, S.M. Gueye, A. NDoye, G. Wotto-Gaye, A. Fall, I. Terolbe, P.D. Ndiaye, A. Mensah

The Sarcoidosis or Besnier Boeck Schaumann's disease is a systemic granulomatous disorders. Multiple organs but specially lungs and skin may be affected. We describe an uncommon case of genital localisation in one patient with epididymal sarcoidosis, regarding on the heterogeneous clinic presentations of this affection.

References show differents hypotesis about the pathogenesis : mycobacterial, viruses, imunologic or genetic factors. There are also a race predominance in black population.

The patient was referred to us for testicular pain associated with a palpable epididymal node, subcutaneous thoracic and limbs, painless nodes, and rhinorrea. With the first clinic approach we attempted the diagnosis of epididymal tuberculosis, which was corrected after to sarcoidosis with the conjunction of several items on a score disease basis. The biopsy confirmed the diagnosis of sarcoidosis, so we began the medical treatment including chloroquine : $300 \mathrm{mg} /$ day during 3 years (to avoid recurrences). As the usual surgical treatment, we submitted our patient to an epididymal node resection. After a 4 years follow-up, there are no evidences of disease.

Key Words : epididymis, sarcoidosis. 